The morning and evening record may be stated as follows: Day. T. (F.) P. R.

a.m. 92 2i Quinin and iron siven.

p. m. $101.8 \quad 98 \quad 2 \overline{5}$ Quinin and iron continued. Complaint

4 a. m. 101.t $100 \quad 24$ Expectoration less. Quinin withdrawn.

a. m. 101.t 10024 Expectoration less. Quinin withdrawn.
Iron continued.

p. m. 16210826 Quinin repeated, gr. xl, followed by gr. xxx in one hour. Iron continued

5
a. m. $\quad 99 \quad 100 \quad 27 \begin{gathered}\text { Quinin and iron both discontinued, owing } \\ \text { to irritability of the stomach. Patient }\end{gathered}$ was not seen in the evening.

6 a.m. $100 \quad 96 \quad 17$ Feeling better. No medicine given.

p. m. $99.4 \quad 100$ 16 Strych. gr. 1/60 every three hour's while p. n. 2.4 awake. Practicaliy no expectoration.

7 a.m. $\quad 98.6 \quad 98 \quad 17$ Strych, gr. 1/60 t. 1. d. with tr. nux et gent, iñ $M . \nabla$. before each meal. reathitions regunds and only adventitions sounds and paing mucous expectoration. No dulness on percussion.

The temperature, pulse and respiration have remained normal, and the patient feels well, though weak. (Eighth day.)

I might add that I did not give such large doses as suggested by Dr. Galbraith; owing to a very irritable stomach. She complained a good deal of tinnitus, but not after quinin was withdrawn.

I would like to ask if people in the southern states are more tolerant of quinin, or if this patient would probably have retained a larger initial close better than the smaller, and if the effect would have been more permanent.

\section{AN IMPROVED RECTAL OPERATING TABLE.}

\section{I. LEFEVRE, M.D.} CLEVELAND, OHIO.

Since publishing a description of my table in the Hedical Record several years ago the table has been remodeled and improved as shown in the following illustrations: Figure 1 shows a front view of the table. Figure 2 shows a back view with the side shelf folded down and the leg rests up in position. The leg rests are adjustable as to height, the padded pieces being hinged on the round supports so that they automatically adjust themselves to the leg, thus assuring a comfortable position. At the same time the buttocks are brought

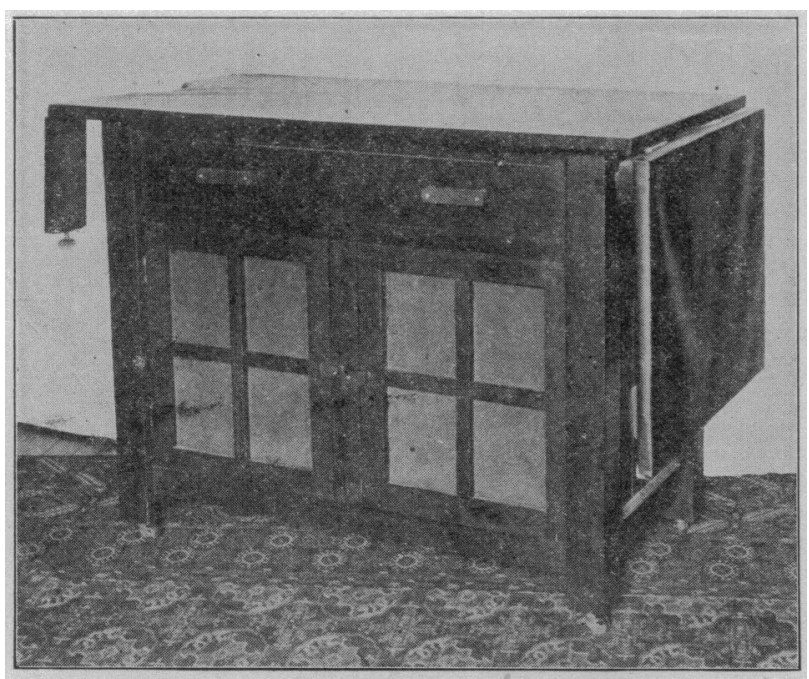

Figure 1

to the end of the table, making the parts easy of access for the operator. When the side shelf is adjusted and locked in position, as shown in Figure 3, and the top tipped to an angle of 90 degrees, the patient is then in position for a proctoscopic exam. ination. With the patient in the Sims position the side shelf can be adjusted and a practically knee-chest position secured, with little or no discomfort to the patient. This is particularly desirable when examining a woman.

While the table is designed especially for rectal work, it is suitable for all ordinary office examinations.

708-712 Rose Building.

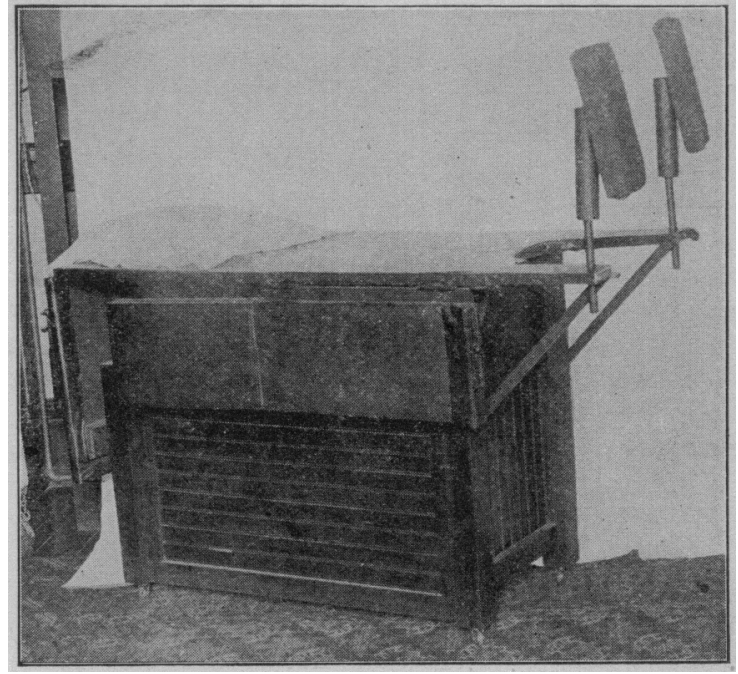

Figure 2.

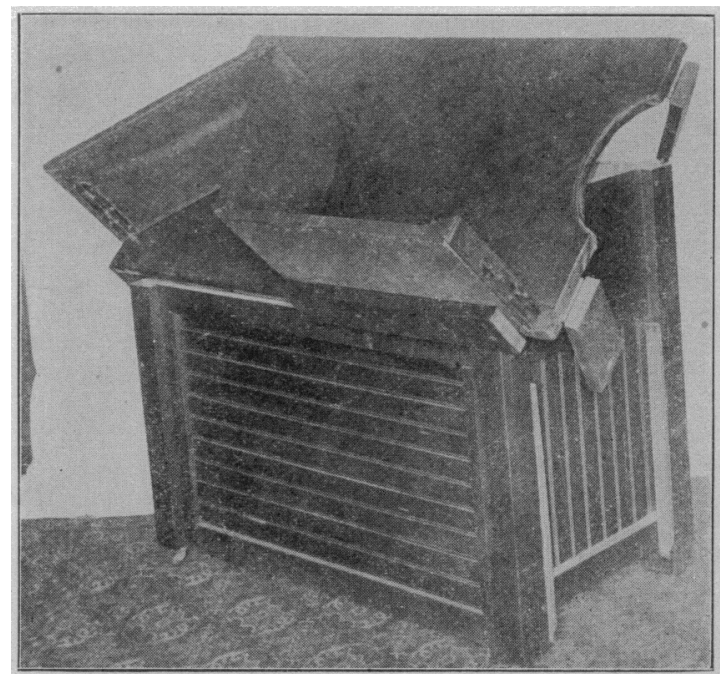

Figure 3.

LOCATING FISTULAE IN THE LOWER INTESTINAL TRACT BY INJECTING HYDROGEN DIOXID THROUGH THE ANUS.*

H. A. ROYSTER, A.B., M.D.

L'rofessor of Gynecology and Dean of the Faculty, Medical Depart ment of the Vniversity of North Carolina: Gynecologist to Iiex Hospital; Surgeon-in-Chief to st. Agnes llospital; Surgeon to the Soutbern Railway. RALEIGII, N. C.

It occasionally happens that in operating for the closure of a fecal fistula the surgeon encounters much difficulty, or even failure, in finding the aperture in the bowel. Especially is this likely to occur when the field of operation is a network of adhesions and the intestinal opening is obscured by blood. To illustrate such a difficulty and to suggest, if possible, a way out of it, I report the following case, in which the technic, so far as known, was original.

Patient.-In 1903, Miss J., aged 22, had a bysterectomy performed. During this operation the sigmoid flexure was injured, and, though the abdominal wound apparently healed, after a

- Read by invitation before the South Carolina Medical Association, Columbla, April 18, 1906 
vear there appeared a small opening in the lower end through which was discharged pus and fecal matter. This persisted for several months, with no tendency to close under the usual methods of treatment. In the latter part of 1904, I was called to see patient in consultation. At this time she was bed-ridden, emaciated, anemic and required rather large doses of morphin to relieve what seemed to be severe pain. A fistulous tract led from the lowest part of the abdominal incision backward, downward and toward the left to a depth of six or seven inches, and according to the patient and her attendants, pus was now and then discharged through the rectum.

Operation.-Jan. 28, 1905, I opened her abdomen in the median line and followed the sinus down to a dense mass of adhesions, which it was impossible to separate without doing damage to the local parts or perilously prolonging an operation on a patient already exhausted. A cigarette drain was introduced and the wound closed around it.

Postoperative History.-At first some hope was entertained that this procedure would have favorable result; but after some weeks the sinus broke down and the fistula again began to discharge. For the next few months the patient was given most careful attention. Her wound was dressed frequently, the diet regulated, tonics administered; in short, everything was done to bring up her nutrition to the standard, with the result that after six months she was in first-rate condition, ready and willing to undergo another operation.

Second Operation.-Accordingly, on Aug. 16, 1905, she was etherized and the abdomen opened. This time I made an incision, much longer than the former one, at the outer border of the left rectus, instead of through the linea alba, thereby obtaining a better view of the structures to be operated on. Disregarding the old sinus, a line of cleavage was made through the adhesions down on the sigmoid flexure, exposing it clearly for some distance above and below the point where the fistula was supposed to be. Oozing from the torn tissues was annoying, but not excessive. All attempts to find the hole in the bowel were at first futile. There was reason to believe it was present; gas could be heard to escape through it and the fecal odor was unmistakable, but it could not be seen. Finally, with the abdomen still open and the affected area in sight, I suddenly thought of the following plan, which was successfully accomplished: I had the nurse pass a long soft rubber catheter into the rectum from below and inject through it one ounce of hydrogen dioxid. As soon as this substance came to the opening in the sigmoid there was an effervescence, plainly discernible, definitely indicating the fistula, the edges of which were immediately grasped with forceps. A purse-string suture of fine silk was placed around the opening, and over this two rows of Lembert sutures, also silk, were applied, the first interrupted and the second continuous. The external incision was closed except for a very thin gauze tape about its middle. The patient's recovery, otherwise uneventful, was complicated by an acute pyelitis on the twelfth day, which threatened to become of serious import. Discussion of the cause of this infection would be interesting, but out of place.

Result.-The patient is now entirely well, having gained steadily in weight since two months after the operation.

From the evidence at hand, there is no recorded case in which the exact method employed by me has been used. A painstaking search of the literature on the subject of fecal fistula in the lower bowel reveals but one instance of even a like character. In 1894, Nicholas Senn $^{1}$ described a very similar operation. His patient was a young woman, 25 years old, who dated her trouble from childbirth, five years and a half before. An abscess had formed in the left ischiorectal space three years afterward.

There was an opening in the gluteal region two inches from the anus and a second opening, which appeared six months later than the first, in the left inguinal region. The patient was brought into Senn's clinic as a case of intestinal fistula. Before the operation, dioxid of hydro-

\footnotetext{
1. Amer. Jour, of Obstetrics, vol. xxx, p. $\mathbf{3 3 5}$.
}

gen was injected through the inguinal fistula and was followed immediately by the escape of white foam from an opening in the rectum, which could be plainly seen through a rectal speculum. The same phenomenon was observed after a similar injection into the gluteal opening, showing that both abscess cavities communicated with the same intestinal fistula. A satisfactory operation was performed through the abdomen.

\section{THE SIMPLEST, EASIEST AND BEST METHOD OF IRRIGATING THE BLADDER.}

\author{
J. D. SOUTHARD, M.D. \\ Surgeon to Belle Point Hospital. \\ FORT SMITH, ARK.
}

Insert one end of a glass pipette into the open end of a soft rubber catheter, over the other end of the pipette slip a piece of rubber tubing. Prepare the irrigating solution in a graduate of proper size. Introduce the catheter and allow the bladder contents to flow out into a convenient vessel; grasp the rubber tubing with left thumb and index finger just as the flow ceases and press it firmly together, thus preventing the entrance of air into the bladder. While thus holding the tube insert into it the stem of a glass funnel and elevate it over the abdomen; at the same time, with the right hand, begin pouring tne solution into the funnel, being careful to keep enough in the funnel to prevent the entrance of air. When the desired quantity has been introduced, press the rubber tube again, lower the funnel and allow the bladder contents again to pass into the vessel. Repeat the operation as often as desired, finally withdrawing the catheter and permitting the last portion to be passed by the patient through the urethra, if desirable.

The graduate affords a simple and accurate means of preparing the solution to be used, and at a glance at any time tells just how much has been introduced.

The solution flows into the bladder in a gentle continuous stream. The apparatus is simplicity itself, easily sterilized and always ready. I believe a trial of it and comparison with other methods will sustain my claim, that it is the simplest, easiest and best.

101 North Sixth Street.

\section{ATTACKS OF HYSTERICAL APHONIA OCCUR- RING IN A PATIENT SUBJECT TO TYPI- CAL EPILEPTIC SEIZURES. THEODORE DILIJER, M.D. PITTSBUR日.}

Patient.-An unmarried woman, aged 26, clerk in postoffice, family history negative.

History.-Since August, 1901, she has been subject to attacks of epilepsy of the ordinary type which at first occurred about once in three months but which have occurred once or twice a month during the past year.

Besides these epileptic fits, the patient has been subject for 3 or 4 years past to frequent attacks of mutism and to attacks consisting of confusion of speech. The last named are often preceded by the first named, i. e., an attack of mutism is often 